\title{
Revenue allocation in Formula One: A pairwise comparison approach
}

\author{
Dóra Gréta Petróczya $\quad$ László Csatób
}

16th December 2020

"The analysis of revenue sharing has paid little attention to the different ways that revenues for sharing can be collected or the basis of their allocation." (Szymanski, 2003, p. 1182)

\begin{abstract}
A model is proposed to allocate Formula One World Championship prize money among the constructors. The methodology is based on pairwise comparison matrices, allows for the use of any weighting method, and makes possible to tune the level of inequality. We introduce an axiom called scale invariance, which requires the ranking of the teams to be independent of the parameter controlling inequality. The eigenvector method is revealed to violate this condition in our dataset, while the row geometric mean method always satisfies it. The revenue allocation is not influenced by the arbitrary valuation given to the race prizes in the official points scoring system of Formula One and takes the intensity of pairwise preferences into account, contrary to the standard Condorcet method. Our approach can be used to share revenues among groups when group members are ranked several times.
\end{abstract}

Keywords: Decision analysis; pairwise comparisons; revenue allocation; Formula One; axiomatic approach

MSC class: 62F07, 90B50, 91B08

JEL classification number: C44, D71

${ }^{a}$ E-mail: doragreta.petroczy@uni-corvinus.hu

Corvinus University of Budapest (BCE), Department of Finance, Budapest, Hungary

b Corresponding author. E-mail: laszlo.csato@sztaki.hu

Institute for Computer Science and Control (SZTAKI), Laboratory on Engineering and Management Intelligence, Research Group of Operations Research and Decision Systems, Budapest, Hungary

Corvinus University of Budapest (BCE), Department of Operations Research and Actuarial Sciences, Budapest, Hungary 


\section{Introduction}

Professional sports leagues and championships generate billions of euros in common revenue. However, its allocation among the participants is often burdened with serious legal disputes centred around unequal shares and the possible violation of competition laws. Consequently, constructing allocation rules which depend only on a few arbitrary variables, and are relatively simple, robust, and understandable for all participants, poses an important topic of academic research.

Formula One (Formula 1, or simply F1) is the highest class of single-seater car racing. A Formula One season consists of several races taking place around the world. The drivers and constructors accumulate points on each race to obtain the annual World Championships, one for drivers, and one for constructors. Since running a team costs at least 100 million US dollars per year, ${ }^{1}$ the distribution of Formula One prize money (1,004 million US dollars in $\left.2019^{2}\right)$ can substantially affect competitive balance and the uncertainty around the expected outcome of races.

Table 1: Formula One prize money allocation, 2019 (in million US dollars)

\begin{tabular}{lcccccc}
\hline Team & Column 1 & Column 2 & $\begin{array}{c}\text { Long-standing } \\
\text { team }\end{array}$ & $\begin{array}{c}\text { Championship } \\
\text { bonus }\end{array}$ & Other & Sum \\
\hline Ferrari & 35 & 56 & 73 & 41 & - & $\mathbf{2 0 5}$ \\
Mercedes & 35 & 66 & - & 41 & 35 & $\mathbf{1 7 7}$ \\
Red Bull & 35 & 46 & - & 36 & 35 & $\mathbf{1 5 2}$ \\
McLaren & 35 & 32 & - & 33 & - & $\mathbf{1 0 0}$ \\
Renault & 35 & 38 & - & - & - & $\mathbf{7 3}$ \\
Haas & 35 & 35 & - & - & - & $\mathbf{7 0}$ \\
Williams & 35 & 15 & - & - & 10 & $\mathbf{6 0}$ \\
Racing Point & 35 & 24 & - & - & - & $\mathbf{5 9}$ \\
Sauber & 35 & 21 & - & - & - & $\mathbf{5 2}$ \\
Toro Rosso & 35 & 17 & - & 151 & 80 & $\mathbf{1 0 0 4}$ \\
\hline Sum & 350 & 350 & 73 & - & - & - \\
\hline
\end{tabular}

Source: https://www.racefans.net/2019/03/03/formula-1-teams-prize-money-paymentsfor-2019-revealed/

The current prize money allocation of Formula One is presented in Table 1. Column 1 corresponds to the revenue distributed equally among the teams which have finished in the top ten in at least two of the past three seasons. Column 2 corresponds to the performance-based payment, determined by the team's finishing position in the previous season. Ferrari has a special Long-standing Team payment as being the only team that competes since the beginning of the championship. Column 4 is paid to the previous champions, and three other teams receive bonus payments.

It is worth noting that only the third of the money pot is allocated strictly on the performance in the previous season and bottom teams are underrepresented. Therefore,

1 The study "Revealed: The $\$ 2.6$ billion budget that fuels F1's 10 teams" by Christian Sylt is available at https://www.forbes.com/sites/csylt/2018/04/08/revealed-the-2-6-billionbudget-that-fuels-f1s-ten-teams.

${ }^{2}$ See the summary titled "Formula 1 teams' prize money payments for 2019 revealed" by Dieter Rencken and Keith Collantine, which can be accessed at https://www.racefans.net/2019/03/03/formula-1teams-prize-money-payments-for-2019-revealed/. 
Table 2: Alternative results of the 2013 Formula One World Championship

(a) Points scoring systems

\begin{tabular}{lcccccccccc}
\hline Year & \multicolumn{1}{c}{ Positions } \\
& 1 & 2 & 3 & 4 & 5 & 6 & 7 & 8 & 9 & 10 \\
\hline $1961-1990$ & 9 & 6 & 4 & 3 & 2 & 1 & 0 & 0 & 0 & 0 \\
$1991-2002$ & 10 & 6 & 4 & 3 & 2 & 1 & 0 & 0 & 0 & 0 \\
$2003-2009$ & 10 & 8 & 6 & 5 & 4 & 3 & 2 & 1 & 0 & 0 \\
$2010-$ & 25 & 18 & 15 & 12 & 10 & 8 & 6 & 4 & 2 & 1 \\
\hline
\end{tabular}

(b) World Constructors' Championship standings

\begin{tabular}{|c|c|c|c|c|c|c|c|c|}
\hline \multirow[t]{3}{*}{ Team } & \multicolumn{8}{|c|}{ Points scoring systems } \\
\hline & \multicolumn{2}{|c|}{ 1961-1990 } & \multicolumn{2}{|c|}{ 1991-2002 } & \multicolumn{2}{|c|}{ 2003-2009 } & \multicolumn{2}{|c|}{ 2010- } \\
\hline & Points & Rank & Points & Rank & Points & Rank & Points & Rank \\
\hline Red Bull & 190 & 1 & 203 & 1 & 243 & 1 & 596 & 1 \\
\hline Mercedes & 88 & 2 & 91 & 2 & 140 & 3 & 360 & 2 \\
\hline Ferrari & 87 & 3 & 89 & 3 & 141 & 2 & 354 & 3 \\
\hline Lotus & 84 & 4 & 85 & 4 & 127 & 4 & 315 & 4 \\
\hline McLaren & 12 & 5 & 12 & 5 & 38 & 5 & 122 & 5 \\
\hline Force India & 6 & 7 & 6 & 7 & 23 & 6 & 77 & 6 \\
\hline Sauber & 7 & 6 & 7 & 6 & 19 & 7 & 57 & 7 \\
\hline Toro Rosso & 1 & 8 & 1 & 8 & 9 & 8 & 33 & 8 \\
\hline Williams & 0 & 9 & 0 & 9 & 1 & 9 & 5 & 9 \\
\hline Marussia & 0 & 10 & 0 & 10 & 0 & 10 & 0 & 10 \\
\hline Caterham & 0 & 11 & 0 & 11 & 0 & 11 & 0 & 11 \\
\hline
\end{tabular}

the new owner of the company controlling Formula One since January 2017 (Liberty Media), plans to reform the revenue allocation of the championship, mainly to increase the competitiveness of smaller teams. ${ }^{3}$

Nonetheless, since the money allocation is based on the ranking of the constructors, it is important to apply a robust and reliable ranking procedure - but the current points scoring system fails to satisfy this criterion. An illustration is provided by Table 2: in the 2013 season, Ferrari would have obtained the second position ahead of Mercedes if the previous points scoring system (effective from 2003 to 2009) would have been used, and Sauber would have been the sixth instead of Force India according to the 1961-1990 and 1991-2002 rules.

The long list of Formula One World Championship points scoring systems, available at https://en.wikipedia.org/wiki/List_of_Formula_One_World_Championship_points_ scoring_systems, highlights the arbitrariness of the points scoring systems, and suggests that the relative importance of the different positions in a race remains unclear.

\footnotetext{
${ }^{3}$ Consider the article "F1 2021: Liberty's masterplan for Formula One's future uncovered" by $D i$ eter Rencken and Keith Collantine, available at https://www.racefans.net/2018/04/11/f1-2021libertys-masterplan-for-formula-ones-future-uncovered/. Another column titled "Revealed: The winners and losers under Liberty's 2021 F1 prize money plan" by Dieter Rencken and Keith Collantine discusses the potential impacts of this plan and can be accessed at https://www.racefans.net/2018/ 04/11/revealed-the-winners-and-losers-under-libertys-2021-f1-prize-money-plan/.
} 
The criticism in Haigh (2009, Section 2) is also worth studying. A website (https: //www. formula1points.com/simulator) dedicated to trialling arbitrary points system definitions shows the popularity of such what-if scenarios among the fans.

Inspired by similar discrepancies, the current paper aims to outline a formal model that can be used to share Formula One prize money among the teams in a meaningful way. Our proposal is based on pairwise comparisons and has strong links to the Analytic Hierarchy Process (AHP), a famous decision-making framework. In particular, we construct a multiplicative pairwise comparison matrix from the race results. Contrary to the Condorcet-like methods (Soares de Mello et al., 2015), it is not only said that a team is preferred to another if it has better results in the majority of the races, but the intensity of these pairwise preferences are also taken into account. Two popular weighting methods, the eigenvector method, and the row geometric mean, are considered to compute the revenue share of each team.

The values of the pairwise comparison matrix depend on a parameter, which is shown to control the inequality of the allocation. Since the ranking of the teams by the eigenvector method is not independent of this parameter, the row geometric mean method has more favourable axiomatic properties in our setting. The application presented in the paper gives some insight into how the parameter influences the expected inequality of the revenue allocation, thus the decision-maker can fix the rules of the distribution before the competition is conducted.

The main contribution of our study is providing an alternative solution in the Formula One industry, which means innovation in reforming revenue allocation and at the same time applicable in other settings to replicate. The pairwise comparison approach has the following, mainly advantageous, features:

- The derivation of the pairwise comparison matrix from the race results depends on a single variable, which regulates the inequality of the distribution. The user can choose this by taking into account preferences on how much inequality is desirable.

- It allows for the use of any weight deriving methods used in the AHP literature;

- Except for its sole parameter, the methodology is not influenced by any ad hoc decision such as the scores used in the official points system of Formula One.

- It supports the reliable performance of the bottom teams, which seldom score points, therefore the current system awards if they achieve unexpected results, mainly due to extreme events in some races.

Besides that, a reasonable axiom in our setting is introduced for weighting methods, and its violation by the eigenvector method is presented in real data.

The paper proceeds as follows. Section 2 gives an overview of related articles. The methodology is presented in Section 3 and is applied in Section 4 to the Formula One World Constructors' Championship between 2014 and 2018. Section 5 concludes.

\section{Literature review}

Our work is connected to several research fields. First, revenue sharing and its impact on competitive balance is a prominent topic of sports economics. Atkinson et al. (1988) 
examine revenue allocation as an incentive mechanism encouraging the desired behaviour of the teams in a league. Késenne (2000) analyses revenue sharing under the profitand utility-maximising hypothesis, and finds that equality promotes competitive balance. However, revenue sharing can lead to a more uneven contest under reasonable assumptions (Szymanski, 2003; Szymanski and Késenne, 2004). Bergantiños and Moreno-Ternero (2020b) give direct, axiomatic, and game-theoretical foundations for two simple rules used to share the money coming from broadcasting sports leagues among participating teams. Bergantiños and Moreno-Ternero (2020a) introduce axioms formalising alternative ways of allocating the extra revenue obtained from additional viewers.

Second, the proposed method is based on multiplicative pairwise comparison matrices (Saaty, 1977, 1980), thus it continues the applications of the AHP in resource allocation problems (Ramanathan and Ganesh, 1995; Saaty et al., 2007). For example, Ossadnik (1996) extensively uses pairwise comparison matrices to allocate the expected synergies in a merger to the partners. Furthermore, the current paper offers theoretical contributions to this area. In particular, we consider two popular weighting methods and introduce a reasonable axiom called scale invariance, that is, the ranking should be independent of the variable governing the inequality of the allocation. Since it will be investigated whether this requirement is satisfied by the two methods in the case of Formula One results, our paper can be regarded as a companion to Duleba and Moslem (2019), which provides the first examination of another property called Pareto efficiency (Blanquero et al., 2006; Bozóki, 2014; Bozóki and Fülöp, 2018) on real data.

Third, there are some direct applications of pairwise comparison matrices in sports ranking. Csató (2013) and Csató (2017) recommend this approach to obtain a fair ranking in Swiss-system chess team tournaments. Bozóki et al. (2016) address the issue of ranking top tennis players of all time, while Chao et al. (2018) evaluate historical Go players.

Fourth, many articles have studied economic problems emerging in Formula One. Rule changes seem to reduce the teams' performances but to improve the competitive balance, and the revenue gain from the latter turns out to be bigger than revenue loss from the former (Mastromarco and Runkel, 2009). Judde et al. (2013) undertake an econometric analysis of competitive balance in this sport. Zaksaitè and Raduševičius (2017) examine the legal aspects of team orders and other tactical decisions in Formula One. According to Budzinski and Müller-Kock (2018), the revenue allocation scheme of this sport is consistent with an anticompetitive interpretation and may be subject to an antitrust investigation. Gutiérrez and Lozano (2020) propose a framework for the design efficiency assessment of some racing circuits that hosted Formula One. Henderson and Kirrane (2018) offer a Bayesian approach to forecast race results. Schreyer and Torgler (2018) investigate whether race outcome uncertainty affects the TV demand for Formula One in Germany and conclude that a balanced competition increases the number of viewers.

Fifth, our procedure leads to an alternative ranking of Formula One constructors, which has its antecedents, too. Kladroba (2000) introduces well-known methods of aggregation to determine the World Championship in 1998. Some of the ranking problems that occurred in the history of Formula One are found to result from defects of the Borda method (Soares de Mello et al., 2005). Haigh (2009) illustrates the instability of the scoring system and argues that any system should be robust to plausible changes, which is not satisfied by the Formula One scoring rules. According to Anderson (2014), subjective point-based rankings may fail to provide an accurate ranking of competitors based on ability. Soares de Mello et al. (2015) present a variant of the Condorcet method with weakly rational decision-makers to compare the teams which competed in the 2013 season. Sitarz (2013) presents the 
incenter of a convex cone to obtain a new ranking of Formula One drivers. Phillips (2014) measures driver performances by adjusting for team and competition effects. Bell et al. (2016) aim to identify the best Formula One drivers of all time. Corvalan (2018) addresses the problem of how the election of the world champion depends on the particular valuation given to the race prizes. Kondratev et al. (2019) characterise the family of geometric scoring rules by the axioms of consistency for adding/removing a unanimous winner and a unanimous loser, respectively. Dietzenbacher and Kondratev (2020) is probably the first paper that develops and motivates prize allocation rules for competitions directly from axioms.

\section{Theoretical background}

In this section, the main components of the model will be presented: the multiplicative pairwise comparison matrix, its derivation from the race results, a straightforward axiom in our setting, and a basic measure of inequality.

\subsection{Multiplicative pairwise comparison matrices}

Consider a set of alternatives $N=\{1,2, \ldots, n\}$ such that their pairwise comparisons are known: $a_{i j}$ shows how many times alternative $i$ is better than alternative $j$.

The sets of positive (with all elements greater than zero) vectors of size $n$ and matrices of size $n \times n$ are denoted by the symbols $\mathbb{R}_{+}^{n}$ and $\mathbb{R}_{+}^{n \times n}$, respectively.

The pairwise comparisons are collected into a matrix satisfying the reciprocity condition, hence any entry below the diagonal equals the reciprocal of the corresponding entry above the diagonal.

Definition 3.1. Multiplicative pairwise comparison matrix: Matrix $\mathbf{A}=\left[a_{i j}\right] \in \mathbb{R}_{+}^{n \times n}$ is a multiplicative pairwise comparison matrix if $a_{j i}=1 / a_{i j}$ holds for all $1 \leq i, j \leq n$.

We will sometimes omit the word "multiplicative" for the sake of simplicity.

Let $\mathcal{A}^{n \times n}$ be the set of pairwise comparison matrices with $n$ alternatives.

Pairwise comparisons are usually used to obtain an approximation of the relative priorities of the alternatives.

Definition 3.2. Weight vector: Vector $\mathbf{w}=\left[w_{i}\right] \in \mathbb{R}_{+}^{n}$ is a weight vector if $\sum_{i=1}^{n} w_{i}=1$.

Let $\mathcal{R}^{n}$ be the set of weight vectors of size $n$.

Definition 3.3. Weighting method: Mapping $f: \mathcal{A}^{n \times n} \rightarrow \mathcal{R}^{n}$ is a weighting method.

The weight of alternative $i$ in the pairwise comparison matrix $\mathbf{A} \in \mathcal{A}^{n \times n}$ according to the weighting method $f$ is denoted by $f_{i}(\mathbf{A})$.

There exist several methods to derive a weight vector, see, for example, Choo and Wedley (2004) for a thorough overview. The most popular procedures are the eigenvector method (Saaty, 1977, 1980), and the row geometric mean (logarithmic least squares) method (Williams and Crawford, 1980; Crawford and Williams, 1985; De Graan, 1980; de Jong, 1984; Rabinowitz, 1976). 
Definition 3.4. Eigenvector method $(E M)$ : The weight vector $\mathbf{w}^{E M}(\mathbf{A}) \in \mathcal{R}^{n}$ provided by the eigenvector method is the solution of the following system of linear equations for any pairwise comparison matrix $\mathrm{A} \in \mathcal{A}^{n \times n}$ :

$$
\mathbf{A} \mathbf{w}^{E M}(\mathbf{A})=\lambda_{\max } \mathbf{w}^{E M}(\mathbf{A}),
$$

where $\lambda_{\max }$ denotes the maximal eigenvalue, also known as the principal or Perron eigenvalue, of the (positive) matrix $\mathbf{A}$.

Definition 3.5. Row geometric mean method (RGM): The row geometric mean method is the function $\mathbf{A} \rightarrow \mathbf{w}^{R G M}(\mathbf{A})$ such that the weight vector $\mathbf{w}^{R G M}(\mathbf{A})$ is given by

$$
w_{i}^{R G M}(\mathbf{A})=\frac{\prod_{j=1}^{n} a_{i j}^{1 / n}}{\sum_{k=1}^{n} \prod_{j=1}^{n} a_{k j}^{1 / n}} .
$$

The row geometric mean method is sometimes called the Logarithmic Least Squares Method $(L L S M)$ because it is the solution to the following optimisation problem:

$$
\min _{\mathbf{w} \in \mathcal{R}^{n}} \sum_{i=1}^{n} \sum_{j=1}^{n}\left[\log a_{i j}-\log \left(\frac{w_{i}}{w_{j}}\right)\right]^{2} .
$$

Although the application of the row geometric mean is axiomatically well-founded (Fichtner, 1986; Barzilai, 1997; Lundy et al., 2017; Bozóki and Tsyganok, 2019), and the eigenvector method has some serious theoretical shortcomings (Johnson et al., 1979; Blanquero et al., 2006; Bana e Costa and Vansnick, 2008), Saaty's proposal remains the default choice of most practitioners. Therefore, both procedures will be considered.

\subsection{From race results to a pairwise comparison matrix}

A Formula One season consists of a series of races, contested by two cars/drivers of each constructor/team. We say that team $i$ has scored one goal against team $j$ if a given car of team $i$ is ahead of a given car of team $j$ in a race. Thus, if there are no incomparable cars, then:

- Team $i(j)$ has scored four (zero) goals against team $j(i)$ if both cars of team $i$ have finished above both cars of team $j$;

- Team $i(j)$ has scored three (one) goal(s) against team $j(i)$ if one car of team $i$ has finished above both cars of team $j$, and the other car of team $i$ has finished above one car of team $j$;

- Team $i(j)$ has scored two (two) goals against team $j(i)$ if one car of team $i$ has finished above both cars of team $j$ but both cars of team $j$ have finished above the other car of team $i{ }^{4}$

The goals scored by the constructors in a race are aggregated over the whole season without weighting, similarly to the official points scoring system. ${ }^{5}$

\footnotetext{
${ }^{4}$ We follow the official definition in classifying a driver as finished if he completed over $90 \%$ of the race distance.

${ }^{5}$ Except for the 2014 Abu Dhabi Grand Prix, which awarded double points as the last race of the season.
} 
Consequently, the maximum number of goals that a team can score against another is four times the number of races. Since a car might not finish a race, it is assumed that each finishing car is better than another, which fails to finish. Nonetheless, two cars may be incomparable if both of them failed to finish the race. In this case, no goal is scored. The goals of the constructors are collected into the $n \times n$ goals matrix.

The pairwise comparison matrix $\mathbf{A}=\left[a_{i j}\right]$ is obtained from the goals matrix: if constructor $i$ has scored $g_{i j}$ goals against constructor $j$, while constructor $j$ has scored $g_{j i}$ goals against constructor $i$, then $a_{i j}=g_{i j} / g_{j i}$ and $a_{j i}=g_{j i} / g_{i j}$ to guarantee the reciprocity condition.

Theoretically, this procedure is ill-defined because the problem of division by zero is not addressed. However, in our dataset $g_{i j}$ was always positive, in other words, at least one car of every team was ahead of one car of any other team at least in one race during the whole season. Thus the somewhat arbitrary adjustment of zeros in Bozóki et al. (2016) is avoided here. Nonetheless, the potential problem cannot be neglected because it did not appear in our dataset. For us, adding a constant $\varepsilon$ to each element of the goals matrix is an attractive solution as the definition $a_{i j}=\left(g_{i j}+\varepsilon\right) /\left(g_{j i}+\varepsilon\right)$ also mitigates the sharp differences between the top and bottom teams, which greatly depend on whether the drivers of the weak team were "lucky" enough to finish certain races. However, the thorough analysis of this suggestion is left to future research.

As we have mentioned, two weighting methods, the $E M$ and the $R G M$ will be used to derive a weight vector, which directly provides an allocation of the available amount.

The presented procedure does not contain any variable, thus it might lead to an allocation that cannot be tolerated by the decision-maker because of its (in)equality. Hence the definition of the pairwise comparison matrix is modified such that:

$$
a_{i j}=\left(\frac{g_{i j}}{g_{j i}}\right)^{\alpha} \quad \text { and } \quad a_{j i}=\left(\frac{g_{j i}}{g_{i j}}\right)^{\alpha} \quad \text { for all } i, j \in N,
$$

where $\alpha \geq 0$ is a parameter. If $\alpha$ is small, then $\mathbf{A}$ is close to the unit matrix, the weights are almost the same, and the shares remain roughly equal. The effect of $\alpha$ will be further investigated in Section 4.

Note that the proposed model is similar to the Condorcet-like methods, widely used in social choice theory, only at first sight. For example, Soares de Mello et al. (2015) introduces a so-called Condorcet graph, where the nodes represent the Formula One teams, and there is a directed edge from node $i$ to node $j$ if team $i$ is preferred to team $j$, that is, if team $i$ is preferred to team $j$ in the majority of races. Therefore, the Condorcet variants take only the pairwise preferences into account but not their intensities. Unsurprisingly, this loss of information often means that the Condorcet method does not provide a strict ranking, while ties between the revenue share of two teams in our model are less frequent (and does not mean any problem).

\subsection{A natural axiom for weighting methods}

Some papers (Genest et al., 1993; Csató and Rónyai, 2016) examine ordinal pairwise preferences, that is, pairwise comparison matrices with entries of $a$ or $1 / a$. This idea has inspired the following requirement, which is also an adaptation of the property called power invariance (Fichtner, 1984) for the ranking of the alternatives.

Axiom 1. Scale invariance: Let $\mathbf{A}=\left[a_{i j}\right] \in \mathcal{A}^{n \times n}$ be any pairwise comparison matrix and $\alpha>0$ be a (positive) parameter. Let $\mathbf{A}^{(\alpha)}=\left[a_{i j}^{(\alpha)}\right] \in \mathcal{A}^{n \times n}$ be the pairwise comparison 
matrix defined by $a_{i j}^{(\alpha)}=a_{i j}^{\alpha}$. Weighting method $f: \mathcal{A}^{n \times n} \rightarrow \mathcal{R}^{n}$ is called scale invariant if for all $1 \leq i, j \leq n$ :

$$
f_{i}(\mathbf{A}) \geq f_{j}(\mathbf{A}) \Longleftrightarrow f_{i}\left(\mathbf{A}^{(\alpha)}\right) \geq f_{j}\left(\mathbf{A}^{(\alpha)}\right)
$$

Scale invariance implies that the ranking of the alternatives does not change if a different scale is used for pairwise comparisons. For example, when only two verbal expressions, "weakly preferred" and "strongly preferred" are allowed, the ranking should remain the same if these preferences are represented by the values 2 and 3, or 4 and 9, respectively.

In the setting of Section 3.2, scale invariance does not allow the ranking of the teams to depend on the parameter $\alpha$, which seems to be reasonable because the underlying data (the goals matrix) are fixed. In other words, if constructor $i$ receives more money than constructor $j$ under any value of $\alpha$, then it should receive more money for all $\alpha>0$.

Lemma 3.1. The eigenvector method does not satisfy scale invariance.

Proof. It is sufficient to provide a counterexample. See Genest et al. (1993, Example 2.1) and Genest et al. (1993, Figure 1), which are based on an example adapted from Kendall (1955).

The following result has already been mentioned in Genest et al. (1993, p. 581-582).

Lemma 3.2. The row geometric mean method satisfies scale invariance.

Proof. Note that $w_{i}^{R G M} \geq w_{j}^{R G M} \Longleftrightarrow \prod_{k=1}^{n} a_{i k} \geq \prod_{k=1}^{n} a_{j k} \Longleftrightarrow \prod_{k=1}^{n} a_{i k}^{(\alpha)} \geq \prod_{k=1}^{n} a_{j k}^{(\alpha)}$, which immediately verifies the statement.

\subsection{Measuring inequality}

A basic indicator of competition among firms is the Herfindahl-Hirschman index (Hannah and Kay, 1977; Rhoades, 1993; Laine, 1995; Owen et al., 2007).

Definition 3.6. Herfindahl-Hirschman index $(H H I)$ : Let $\mathbf{w} \in \mathcal{R}^{n}$ be a weight vector. Its Herfindahl-Hirschman index is:

$$
H H I(\mathbf{w})=\sum_{i=1}^{n} w_{i}^{2}
$$

The maximum of $H H I$ is one when one constructor receives the whole amount. However, its minimum (reached when all constructors receive the same amount) is influenced by the number of constructors, therefore it is worth considering a normalised version of the $H H I$.

Definition 3.7. Normalised Herfindahl-Hirschman index $\left(H H I^{*}\right)$ : Let $\mathbf{w} \in \mathcal{R}^{n}$ be a weight vector. Its normalised Herfindahl-Hirschman index is:

$$
H H I^{*}(\mathbf{w})=\frac{H H I(\mathbf{w})-1 / n}{1-1 / n}=\frac{\sum_{i=1}^{n} w_{i}^{2}-1 / n}{1-1 / n} .
$$

The value of $H H I^{*}$ is always between 0 (equal shares) and 1 (maximal inequality).

Since the HHI better reflects market concentration, while the normalised HerfindahlHirschman index quantifies the equality of distributions, we will use the latter. 
Table 3: Goals matrix, 2014

\begin{tabular}{|c|c|c|c|c|c|c|c|c|c|c|c|}
\hline & 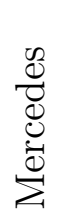 & 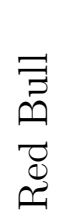 & 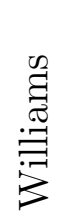 & 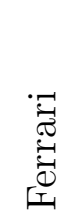 & 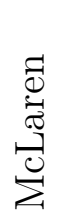 & 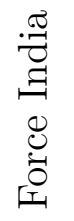 & 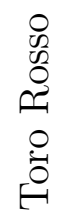 & $\begin{array}{l}n \\
\stackrel{n}{0} \\
\stackrel{0}{0}\end{array}$ & 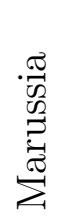 & $\begin{array}{l}\dot{\vec{d}} \\
\frac{0}{\vec{Z}} \\
\tilde{\tilde{D}} \\
\tilde{n}\end{array}$ & 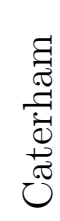 \\
\hline Mercedes & - & 61 & 63 & 62 & 64 & 64 & 65 & 65 & 66 & 66 & 66 \\
\hline Red Bull & 14 & - & 43 & 54 & 56 & 58 & 64 & 64 & 64 & 64 & 64 \\
\hline Williams & 11 & 31 & - & 44 & 47 & 47 & 62 & 66 & 68 & 68 & 68 \\
\hline Ferrari & 13 & 22 & 31 & - & 42 & 48 & 64 & 66 & 69 & 70 & 69 \\
\hline McLaren & 11 & 20 & 29 & 34 & - & 41 & 59 & 65 & 69 & 70 & 71 \\
\hline Force India & 12 & 16 & 28 & 28 & 35 & - & 52 & 61 & 64 & 63 & 64 \\
\hline Toro Rosso & 10 & 7 & 11 & 12 & 16 & 21 & - & 47 & 56 & 54 & 56 \\
\hline Lotus & 5 & 8 & 6 & 10 & 11 & 11 & 22 & - & 49 & 40 & 51 \\
\hline Marussia & 6 & 8 & 7 & 7 & 7 & 7 & 15 & 16 & - & 28 & 34 \\
\hline Sauber & 7 & 8 & 4 & 5 & 2 & 5 & 13 & 29 & 44 & - & 44 \\
\hline Caterham & 3 & 8 & 5 & 6 & 3 & 4 & 15 & 12 & 22 & 23 & - \\
\hline
\end{tabular}

\section{Results}

To illustrate the proposed allocation scheme, the 2014 Formula One season will be investigated in detail. Table 3 shows the goals matrix where the teams are ranked according to the official championship result (Sauber and Marussia both obtained zero points this year). For example, a car of Mercedes was better than a car of Red Bull on 61 occasions, while a car of Red Bull was better than a car of Mercedes on 14 occasions.

The corresponding pairwise comparison is presented in Table 4 with the choice $\alpha=1$. It can be seen that all entries above the diagonal are higher than the corresponding element below the diagonal, thus a team, which has scored more points in the official ranking, is almost always preferred to a team with a lower number of points by pairwise comparisons. The only exception is Marussia vs. Sauber, where the latter constructor has a robust advantage.

Figure 1 plots the shares of the 11 competing teams with our methodology. Due to the same underlying pairwise comparison matrix, results given by the eigenvector and row geometric mean methods do not differ substantially. Nonetheless, Mercedes, McLaren, and Sauber are always better off with the eigenvector method, while Red Bull prefers it if $\alpha$ does not exceed 1.25 .

Mercedes is the dominant team according to both methods, with an ever increasing share as a function of parameter $\alpha$ (see Figure 1.a). There are five middle teams (Red Bull, Williams, Ferrari, McLaren, Force India), which receive more money than the equal share in the case of small $\alpha$ (see Figure 1.b). Their ranking coincides with the official one for the row geometric mean, although Ferrari, which has scored 216 points in the season, has only a negligible advantage over McLaren, which has scored 181 points. On the other hand, the five weakest teams (Toro Rosso, Lotus, Marussia, Sauber, Caterham) receive less if parameter $\alpha$ increases (see Figure 1.c). The row geometric mean ranking reflects the official ranking again, although the difference between the shares of Marussia and Sauber is barely visible.

Lemma 3.2 guarantees that the ranking of the constructors according to their share of 
Table 4: Pairwise comparison matrix, $\alpha=1,2014$

\begin{tabular}{|c|c|c|c|c|c|c|c|c|c|c|c|}
\hline & 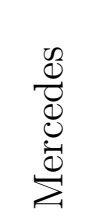 & 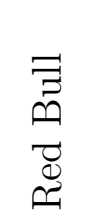 & 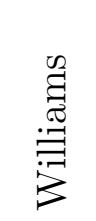 & 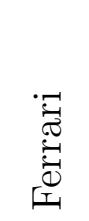 & 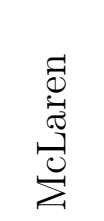 & 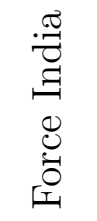 & $\begin{array}{l}0 \\
0 \\
0 \\
0 \\
01 \\
0 \\
0 \\
0 \\
0\end{array}$ & 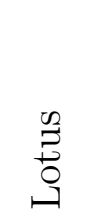 & 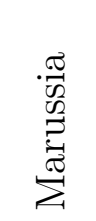 & $\begin{array}{l}\dot{\bar{\Delta}} \\
\stackrel{0}{\Xi} \\
\tilde{\tilde{N}} \\
\tilde{n}\end{array}$ & 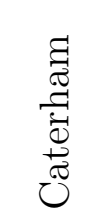 \\
\hline Mercedes & 1 & 4.36 & 5.73 & 4.77 & 5.82 & 5.33 & 6.5 & 13 & 11 & 9.43 & 22 \\
\hline Red Bull & 0.23 & 1 & 1.39 & 2.45 & 2.8 & 3.63 & 9.14 & 8 & 8 & 8 & 8 \\
\hline Williams & 0.17 & 0.72 & 1 & 1.42 & 1.62 & 1.68 & 5.64 & 11 & 9.71 & 17 & 13.6 \\
\hline Ferrari & 0.21 & 0.41 & 0.70 & 1 & 1.24 & 1.71 & 5.33 & 6.6 & 9.86 & 14 & 11.5 \\
\hline McLaren & 0.17 & 0.36 & 0.62 & 0.81 & 1 & 1.17 & 3.69 & 5.91 & 9.86 & 35 & 23.67 \\
\hline Force India & 0.19 & 0.28 & 0.60 & 0.58 & 0.85 & 1 & 2.48 & 5.55 & 9.14 & 12.6 & 16 \\
\hline Toro Rosso & 0.15 & 0.11 & 0.18 & 0.19 & 0.27 & 0.40 & 1 & 2.14 & 3.73 & 4.15 & 3.73 \\
\hline Lotus & 0.08 & 0.13 & 0.09 & 0.15 & 0.17 & 0.18 & 0.47 & 1 & 3.06 & 1.38 & 4.25 \\
\hline Marussia & 0.09 & 0.13 & 0.10 & 0.10 & 0.10 & 0.11 & 0.27 & 0.33 & 1 & 0.64 & 1.55 \\
\hline Sauber & 0.11 & 0.13 & 0.06 & 0.07 & 0.03 & 0.08 & 0.24 & 0.73 & 1.57 & 1 & 1.91 \\
\hline Caterham & 0.05 & 0.13 & 0.07 & 0.09 & 0.04 & 0.06 & 0.27 & 0.24 & 0.65 & 0.52 & 1 \\
\hline
\end{tabular}

the revenue remains unchanged as a function of $\alpha$ if the row geometric mean method is applied. On the contrary, the eigenvector method can lead to a rank reversal: Figure 1.b shows that McLaren will receive a higher share than Williams if $\alpha$ is bigger than 1.5. There are two other changes in the ranking as uncovered by Figure 2: Ferrari receives more money than McLaren, and Marussia receives more money than Sauber for small values of the parameter. Note that row geometric mean always favours the former teams, Ferrari and Marussia.

Thus the violation of scale invariance by the eigenvector method - similarly to the violation of Pareto efficiency (Duleba and Moslem, 2019) - is not only a theoretical curiosity (Lemma 3.1) as real data, in our case Formula One results from the 2014 season, may result in such an undesired rank reversal.

The theoretical explanation of these results remains challenging. It is probably sufficient to cite Herman and Koczkodaj (1996, p. 29): "It is improbable that an analytical solution can be devised in a situation where the results favour GM over EV for one metric while favouring EV over GM for another metric." At least, according to Saaty and Vargas (1984, Theorem 9), both weighting methods satisfy row dominance, namely, for any pair of alternatives $i, j$, if $a_{i k} \geq a_{j k}$ holds for all $k$, then $w_{i} \geq w_{j}$. For example, this means that Force India should receive at least as much money as Toro Rosso (take a look at Table 4). Recently, Kułakowski et al. (2020) have provided lower and upper bounds with the use of the Koczkodaj inconsistency index (Koczkodaj, 1993; Duszak and Koczkodaj, 1994) for the maximal difference of the weights derived by the eigenvector and the geometric mean methods. In addition, the two priority vectors are proved to be more similar if the pairwise comparison matrix is less inconsistent (Kułakowski et al., 2020, Theorem 19), which corresponds to a lower $\alpha$ in our case. A deeper analysis of this issue is the subject of future research.

Table 5 presents the standing in the 2014 season under the four basic historical points scoring systems that are detailed in Table 2.b. The official 2014 scheme, which awarded double points in the last race, is not included, however, it leads to the same ranking as the other four rules. The ranking is identical to the one determined by the row geometric 
Figure 1: Revenue shares, 2014

(a) The top team: Mercedes

Eigenvector method (EM)

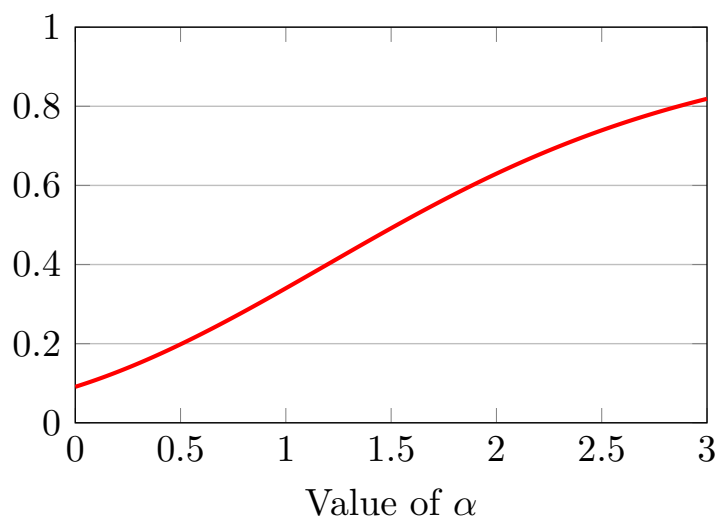

Row geometric mean method (RGM)

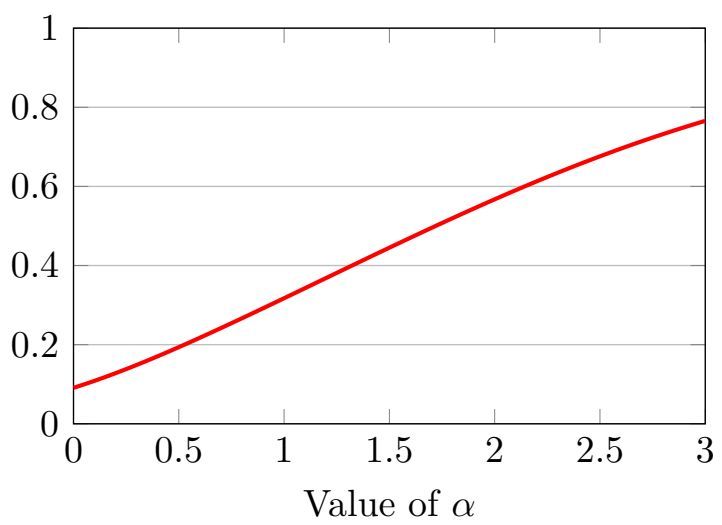

(b) The five middle teams

Eigenvector method $(E M)$

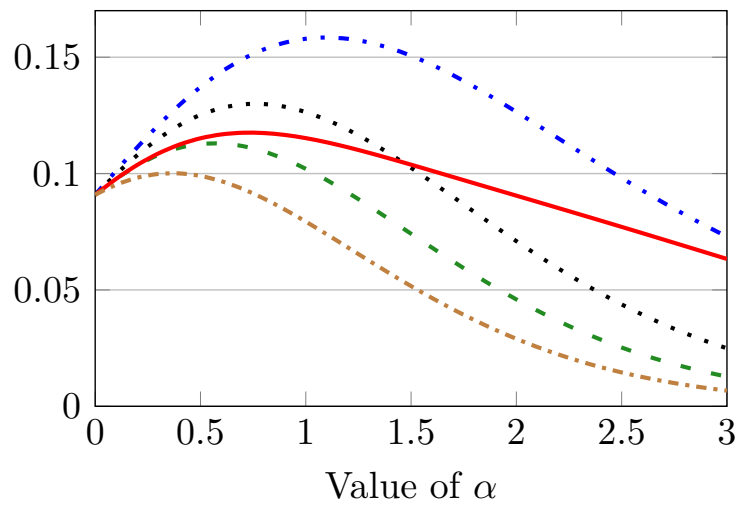

Row geometric mean method ( $R G M)$

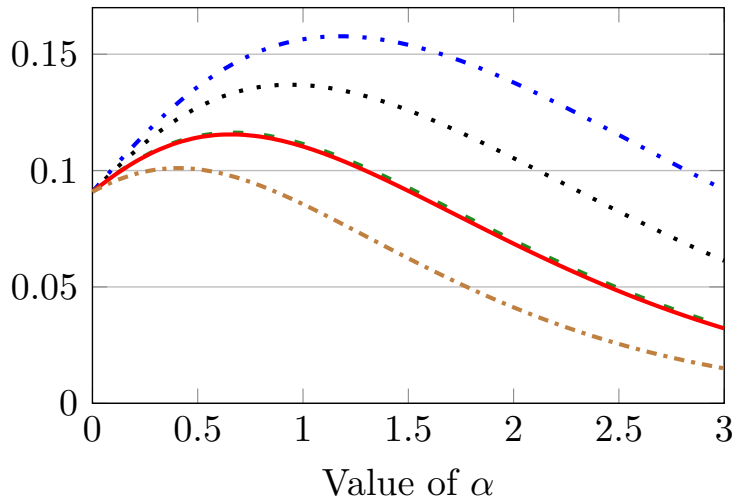

\begin{tabular}{|c|c|c|c|}
\hline - . R Red Bull & ...Williams & - - Ferrari & -.... Force India \\
\hline
\end{tabular}

(c) The five bottom teams

Eigenvector method $(E M)$

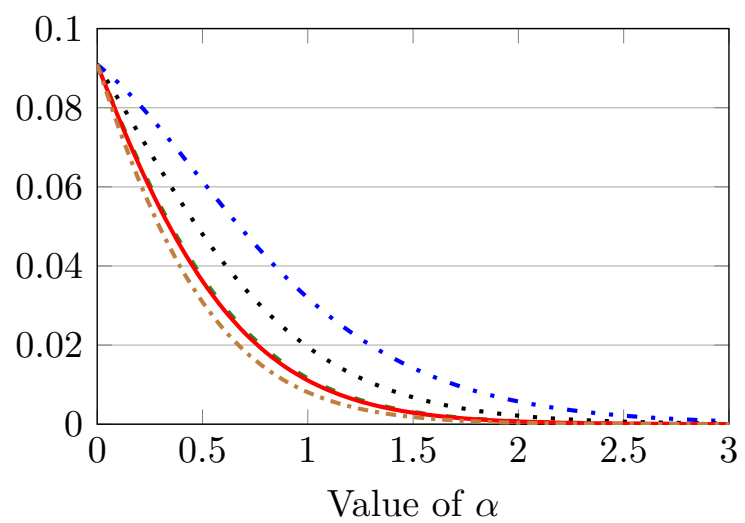

Row geometric mean method ( $R G M)$

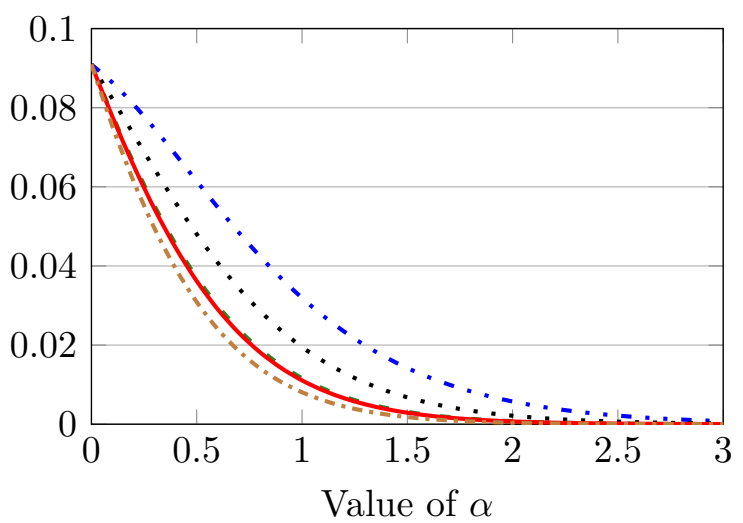

-.. Toro Rosso $\quad \cdots$ Lotus - - Marussia - Sauber -.... Caterham

mean method. This can be another argument against the use of the eigenvector method.

Figure 3 depicts the value of the inequality measure $H H I^{*}$ as a function of parameter 
Figure 2: The difference between the revenue shares of some constructors according to the eigenvector method (EM), 2014
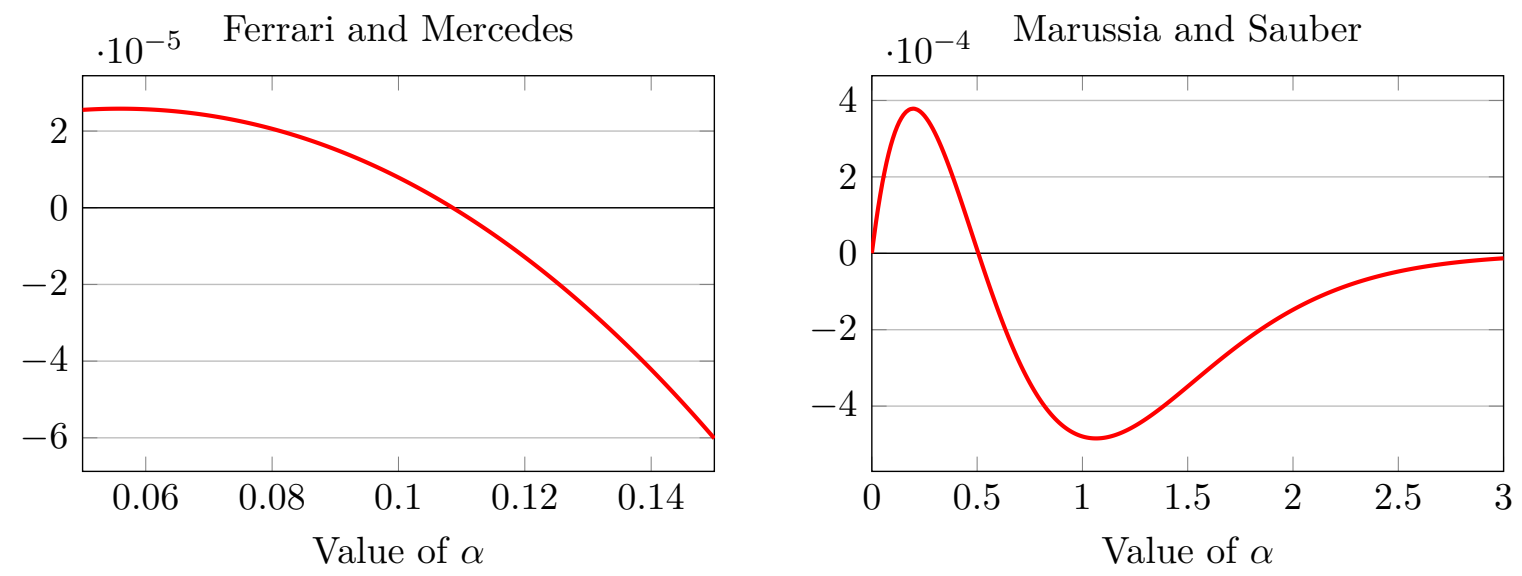

Table 5: Alternative results of the 2014 Formula One World Championship

Team

Points scoring systems

$1961-1990$ 1991-2002

2003-2009

2010-

Points Rank Points Rank Points Rank Points Rank

\begin{tabular}{lcccccccc}
\hline Mercedes & 233 & 1 & 249 & 1 & 281 & 1 & $\mathbf{6 7 6}$ & $\mathbf{1}$ \\
Red Bull & 96 & 2 & 99 & 2 & 154 & 2 & $\mathbf{3 8 9}$ & $\mathbf{2}$ \\
Williams & 62 & 3 & 62 & 3 & 113 & 3 & $\mathbf{2 8 7}$ & $\mathbf{3}$ \\
Ferrari & 36 & 4 & 36 & 4 & 78 & 4 & $\mathbf{2 1 3}$ & $\mathbf{4}$ \\
McLaren & 31 & 5 & 31 & 5 & 62 & 5 & $\mathbf{1 7 1}$ & $\mathbf{5}$ \\
Force India & 16 & 6 & 16 & 6 & 46 & 6 & $\mathbf{1 4 1}$ & $\mathbf{6}$ \\
Toro Rosso & 1 & 7 & 1 & 7 & 5 & 7 & $\mathbf{3 0}$ & $\mathbf{7}$ \\
Lotus & 0 & 8 & 0 & 8 & 2 & 8 & $\mathbf{1 0}$ & $\mathbf{8}$ \\
Marussia & 0 & 9 & 0 & 9 & 0 & 9 & $\mathbf{2}$ & $\mathbf{9}$ \\
Sauber & 0 & 10 & 0 & 10 & 0 & 10 & $\mathbf{0}$ & $\mathbf{1 0}$ \\
Caterham & 0 & 11 & 0 & 11 & 0 & 11 & $\mathbf{0}$ & $\mathbf{1 1}$ \\
\hline
\end{tabular}

$\alpha$ in the five seasons between 2014 and 2018. This can be especially relevant for a decision-maker who should fix the rules before the start of a season with having in mind a maximal level of inequality. For instance, choosing $\alpha=1$ provides that the normalised Herfindahl-Hirschman index will not exceed 0.31 if the given season remains more balanced than the 2017 season.

As our intuition suggests, a higher $\alpha$ results in a more unequal distribution. It is also worth noting that $H H I^{*}$ is consistently smaller in the case of row geometric mean than for the eigenvector method. This can be explained by an important finding of Herman and Koczkodaj (1996, p. 28): "After all, geometric means are 'means', and therefore the Euclidean metric (which 'equalizes' differences of all elements) generates better results for GM than for EV solutions." In other words, the eigenvector method allows for bigger differences between the ratios of the weights, which is - according to Definition 3.6 - unfavourable for $H H I$, thus for $H H I^{*}$, too. Furthermore, while the order of the seasons by the normalised Herfindahl-Hirschman index for a given $\alpha$ is relatively robust, the shape of the five lines varies. 
Figure 3: The normalised Herfindahl-Hirschman index $\left(H H I^{*}\right), 2014-2018$

Eigenvector method $(E M)$

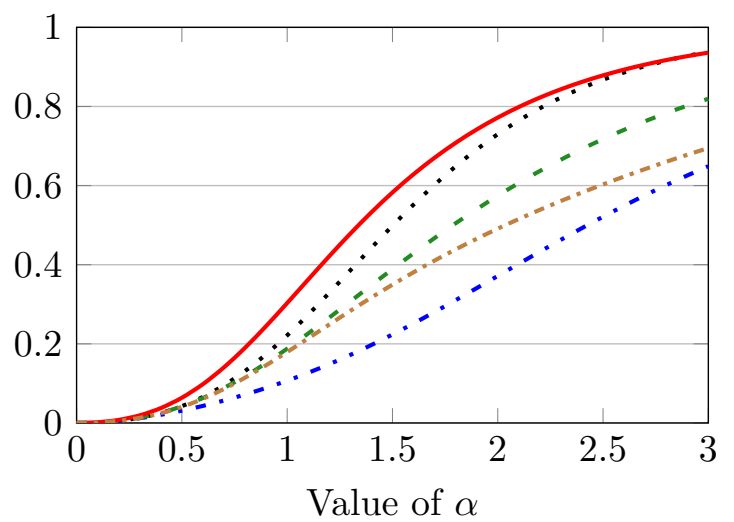

Row geometric mean method $(R G M)$

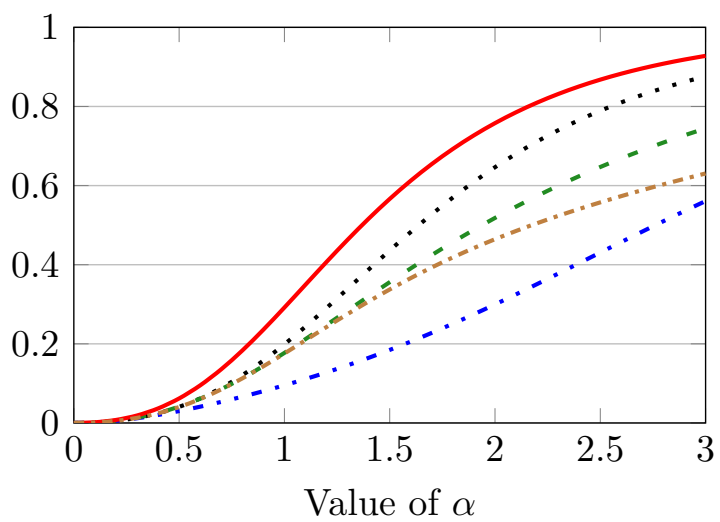

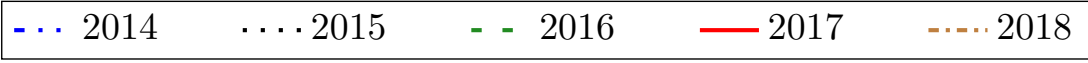

Table 6: Revenue allocation based on performance in the 2018 season

\begin{tabular}{lccccc}
\hline Constructor & Position & $\begin{array}{c}\text { Column 2 revenue } \\
\text { Million US dollars }\end{array}$ & Share (\%) & \multicolumn{2}{c}{ Indifferent $\alpha$} \\
& & 69 & 18.86 & 0.35 & 0.35 \\
\hline Mercedes & 1 & 56 & 16.00 & 0.35 & 0.35 \\
Ferrari & 2 & 46 & 13.14 & - & - \\
Red Bull & 3 & 38 & 10.86 & - & - \\
Renault & 4 & 35 & 10.00 & 0 & 0 \\
Haas & 5 & 32 & 9.14 & 0.13 & 0.14 \\
McLaren & 6 & 24 & 6.86 & 0.6 & 0.61 \\
Racing Point & 7 & 21 & 6.00 & 0.55 & 0.55 \\
Sauber & 8 & 17 & 4.86 & 0.59 & 0.59 \\
Toro Rosso & 9 & 15 & 4.29 & 0.6 & 0.6 \\
Williams & 10 & & & & \\
\hline
\end{tabular}

Column 2 revenue is paid on a sliding scale depending on the teams' finishing position. Source: https://www.racefans .net/2019/03/03/formula-1-teams-prizemoney-payments-for-2019-revealed/

Finally, Table 6 summarises the allocation of revenues in 2019, based on the 2018 season. If the pot would be shared equally, then each team would receive 10 million US dollars. Hence, the constructor Haas is indifferent between its actual position and our proposal with $\alpha=0$. By increasing the parameter, only the three top teams (Mercedes, Ferrari, Red Bull) will receive more money, implying that Renault could not receive its actual share $(10.86 \%)$ under any $\alpha$. The same holds for Red Bull. ${ }^{6}$ Mercedes and Ferrari would be indifferent if $\alpha=0.35$ as the last two columns of Table 5 show. McLaren prefers our proposal until $\alpha$ stands at a relatively small level. The remaining four teams support the current allocation only if $\alpha$ exceeds 0.55 , when the normalised Herfindahl-Hirschman index becomes about 0.05 (see Figure 3). Consequently, the pairwise comparison approach contains some non-linearity: the revenue share of certain teams is a non-monotonic function of the inequality parameter, thus the teams cannot be separated into two sets such that one group prefers a small $\alpha$, and the other benefits from a large $\alpha$.

\footnotetext{
${ }^{6}$ Figure 1.b illustrates that a team might receive its maximal share at a particular $\alpha>0$.
} 


\section{Conclusions}

We have presented a model to share the revenue of an industry through the example of the Formula One World Constructors' Championship. The methodology is based on multiplicative pairwise comparison matrices and makes possible to tune the inequality of the allocation by its single parameter. Since the choice of the weighting method has only a marginal effect in this particular application, we recommend using the row geometric mean, which has favourable theoretical properties. The proposed technique has an important advantage over the official points scoring system of Formula One as it is independent of the somewhat arbitrary valuation given to the race prizes.

Besides offering a novel way for sharing Formula One prize money among the constructors, our methodology can be applied in any area where resources/revenues should be allocated among groups whose members are ranked several times. Potential examples include further racing competitions such as Grand Prix motorcycle racing, combined events in athletics like decathlon and heptathlon, the performance of countries in the Olympic Games, schools on the basis of student tests in various subject areas, or even workplaces where individual contributions on various projects are ranked. The suggested approach is also able to rank Formula One drivers. While this would probably lead to an incomplete pairwise comparison matrix as some pilots cannot be compared, both the eigenvector and the row geometric mean method have been generalised for such matrices (Kwiesielewicz, 1996; Bozóki et al., 2010).

Our paper can inspire further research in various fields. The first possible direction is the analysis of other weighting methods concerning scale invariance. The proposed methodology provides a new ranking of the Formula One constructors, which can be compared to alternative ranking systems. Finally, representing race results in a pairwise comparison matrix may encourage novel ways to measure competitive balance by inconsistency indices (Brunelli, 2018).

\section{Acknowledgements}

We are grateful to Sándor Bozóki and Tamás Halm for useful comments.

Four anonymous reviewers provided valuable remarks and suggestions on earlier drafts. The research was supported by the MTA Premium Postdoctoral Research Program grant PPD2019-9/2019, the NKFIH grant K 128573, and the ÚNKP-19-3-III-BCE-97 New National Excellence Program of the Ministry for Innovation and Technology.

\section{References}

Anderson, A. (2014). Maximum likelihood ranking in racing sports. Applied Economics, 46(15):1778-1787.

Atkinson, S. E., Stanley, L. R., and Tschirhart, J. (1988). Revenue sharing as an incentive in an agency problem: An example from the national football league. The RAND Journal of Economics, 19(1):27-43.

Bana e Costa, C. A. and Vansnick, J.-C. (2008). A critical analysis of the eigenvalue method used to derive priorities in AHP. European Journal of Operational Research, 187(3):1422-1428. 
Barzilai, J. (1997). Deriving weights from pairwise comparison matrices. Journal of the Operational Research Society, 48(12):1226-1232.

Bell, A., Smith, J., Sabel, C. E., and Jones, K. (2016). Formula for success: multilevel modelling of Formula One driver and constructor performance, 1950-2014. Journal of Quantitative Analysis in Sports, 12(2):99-112.

Bergantiños, G. and Moreno-Ternero, J. D. (2020a). Allocating extra revenues from broadcasting sports leagues. Journal of Mathematical Economics, 90(10):65-73.

Bergantiños, G. and Moreno-Ternero, J. D. (2020b). Sharing the revenues from broadcasting sport events. Management Science, 66(6):2417-2431.

Blanquero, R., Carrizosa, E., and Conde, E. (2006). Inferring efficient weights from pairwise comparison matrices. Mathematical Methods of Operations Research, 64(2):271-284.

Bozóki, S. (2014). Inefficient weights from pairwise comparison matrices with arbitrarily small inconsistency. Optimization, 63(12):1893-1901.

Bozóki, S., Csató, L., and Temesi, J. (2016). An application of incomplete pairwise comparison matrices for ranking top tennis players. European Journal of Operational Research, 248(1):211-218.

Bozóki, S. and Fülöp, J. (2018). Efficient weight vectors from pairwise comparison matrices. European Journal of Operational Research, 264(2):419-427.

Bozóki, S., Fülöp, J., and Rónyai, L. (2010). On optimal completion of incomplete pairwise comparison matrices. Mathematical and Computer Modelling, 52(1-2):318-333.

Bozóki, S. and Tsyganok, V. (2019). The (logarithmic) least squares optimality of the arithmetic (geometric) mean of weight vectors calculated from all spanning trees for incomplete additive (multiplicative) pairwise comparison matrices. International Journal of General Systems, 48(4):362-381.

Brunelli, M. (2018). A survey of inconsistency indices for pairwise comparisons. International Journal of General Systems, 47(8):751-771.

Budzinski, O. and Müller-Kock, A. (2018). Is the revenue allocation scheme of Formula One motor racing a case for European competition policy? Contemporary Economic Policy, 36(1):215-233.

Chao, X., Kou, G., Li, T., and Peng, Y. (2018). Jie Ke versus AlphaGo: A ranking approach using decision making method for large-scale data with incomplete information. European Journal of Operational Research, 265(1):239-247.

Choo, E. U. and Wedley, W. C. (2004). A common framework for deriving preference values from pairwise comparison matrices. Computers \& Operations Research, 31(6):893-908.

Corvalan, A. (2018). How to rank rankings? Group performance in multiple-prize contests. Social Choice and Welfare, 51(2):361-380.

Crawford, G. and Williams, C. (1985). A note on the analysis of subjective judgment matrices. Journal of Mathematical Psychology, 29(4):387-405. 
Csató, L. (2013). Ranking by pairwise comparisons for Swiss-system tournaments. Central European Journal of Operations Research, 21(4):783-803.

Csató, L. (2017). On the ranking of a Swiss system chess team tournament. Annals of Operations Research, 254(1-2):17-36.

Csató, L. and Rónyai, L. (2016). Incomplete pairwise comparison matrices and weighting methods. Fundamenta Informaticae, 144(3-4):309-320.

De Graan, J. G. (1980). Extensions of the multiple criteria analysis method of T. L. Saaty. Report, National Institute for Water Supply, Voorburg.

de Jong, P. (1984). A statistical approach to Saaty's scaling method for priorities. Journal of Mathematical Psychology, 28(4):467-478.

Dietzenbacher, B. J. and Kondratev, A. Y. (2020). Fair and consistent prize allocation in competitions. Manuscript. arXiv: 2008.08985.

Duleba, S. and Moslem, S. (2019). Examining Pareto optimality in analytic hierarchy process on real Data: An application in public transport service development. Expert Systems with Applications, 116:21-30.

Duszak, Z. and Koczkodaj, W. W. (1994). Generalization of a new definition of consistency for pairwise comparisons. Information Processing Letters, 52(5):273-276.

Fichtner, J. (1984). Some thoughts about the mathematics of the Analytic Hierarchy Process. Technical report, Institut für Angewandte Systemforschung und Operations Research, Universität der Bundeswehr München.

Fichtner, J. (1986). On deriving priority vectors from matrices of pairwise comparisons. Socio-Economic Planning Sciences, 20(6):341-345.

Genest, C., Lapointe, F., and Drury, S. W. (1993). On a proposal of Jensen for the analysis of ordinal pairwise preferences using Saaty's eigenvector scaling method. Journal of Mathematical Psychology, 37(4):575-610.

Gutiérrez, E. and Lozano, S. (2020). Benchmarking Formula One auto racing circuits: a two stage DEA approach. Operational Research, 20:2059-2083.

Haigh, J. (2009). Uses and limitations of mathematics in sport. IMA Journal of Management Mathematics, 20(2):97-108.

Hannah, L. and Kay, J. A. (1977). Concentration in Modern Industry: Theory, measurement and the U.K. experience. MacMillan, London.

Henderson, D. A. and Kirrane, L. J. (2018). A comparison of truncated and time-weighted Plackett-Luce models for probabilistic forecasting of Formula One results. Bayesian Analysis, 13(2):335-358.

Herman, M. W. and Koczkodaj, W. W. (1996). A Monte Carlo study of parwise comparison. Information Processing Letters, 57(1):25-29.

Johnson, C. R., Beine, W. B., and Wang, T. J. (1979). Right-left asymmetry in an eigenvector ranking procedure. Journal of Mathematical Psychology, 19(1):61-64. 
Judde, C., Booth, R., and Brooks, R. (2013). Second place is first of the losers: An analysis of competitive balance in Formula One. Journal of Sports Economics, 14(4):411-439.

Kendall, M. G. (1955). Further contributions to the theory of paired comparisons. Biometrics, 11(1):43-62.

Késenne, S. (2000). Revenue sharing and competitive balance in professional team sports. Journal of Sports Economics, 1(1):56-65.

Kladroba, A. (2000). Das Aggregationsproblem bei der Erstellung von Rankings. Einige Anmerkungen am Beispiel der Formel 1 Weltmeisterschaft 1998. Jahrbücher für Nationalökonomie und Statistik, 220(3):302-314.

Koczkodaj, W. W. (1993). A new definition of consistency of pairwise comparisons. Mathematical and Computer Modelling, 18(7):79-84.

Kondratev, A. Y., Ianovski, E., and Nesterov, A. S. (2019). How should we score athletes and candidates: geometric scoring rules. Manuscript. arXiv: 1907.05082.

Kułakowski, K., Mazurek, J., and Strada, M. (2020). On the similarity between ranking vectors in the pairwise comparison method. Manuscript. arXiv: 2010.04778.

Kwiesielewicz, M. (1996). The logarithmic least squares and the generalized pseudoinverse in estimating ratios. European Journal of Operational Research, 93(3):611-619.

Laine, C. R. (1995). The Herfindahl-Hirschman index: a concentration measure taking the consumer's point of view. The Antitrust Bulletin, 40(2):423-432.

Lundy, M., Siraj, S., and Greco, S. (2017). The mathematical equivalence of the "spanning tree" and row geometric mean preference vectors and its implications for preference analysis. European Journal of Operational Research, 257(1):197-208.

Mastromarco, C. and Runkel, M. (2009). Rule changes and competitive balance in Formula One motor racing. Applied Economics, 41(23):3003-3014.

Ossadnik, W. (1996). AHP-based synergy allocation to the partners in a merger. European Journal of Operational Research, 88(1):42-49.

Owen, P. D., Ryan, M., and Weatherston, C. R. (2007). Measuring competitive balance in professional team sports using the Herfindahl-Hirschman index. Review of Industrial Organization, 31(4):289-302.

Phillips, A. J. K. (2014). Uncovering Formula One driver performances from 1950 to 2013 by adjusting for team and competition effects. Journal of Quantitative Analysis in Sports, 10(2):261-278.

Rabinowitz, G. (1976). Some comments on measuring world influence. Conflict Management and Peace Science, 2(1):49-55.

Ramanathan, R. and Ganesh, L. S. (1995). Using AHP for resource allocation problems. European Journal of Operational Research, 80(2):410-417.

Rhoades, S. A. (1993). The Herfindahl-Hirschman index. Federal Reserve Bulletin, $79(3): 188-189$. 
Saaty, T. L. (1977). A scaling method for priorities in hierarchical structures. Journal of Mathematical Psychology, 15(3):234-281.

Saaty, T. L. (1980). The Analytic Hierarchy Process: Planning, Priority Setting, Resource Allocation. McGraw-Hill, New York.

Saaty, T. L., Peniwati, K., and Shang, J. S. (2007). The analytic hierarchy process and human resource allocation: Half the story. Mathematical and Computer Modelling, 46(7-8):1041-1053.

Saaty, T. L. and Vargas, L. G. (1984). Comparison of eigenvalue, logarithmic least squares and least squares methods in estimating ratios. Mathematical Modelling, 5(5):309-324.

Schreyer, D. and Torgler, B. (2018). On the role of race outcome uncertainty in the TV demand for Formula 1 Grands Prix. Journal of Sports Economics, 19(2):211-229.

Sitarz, S. (2013). The medal points' incenter for rankings in sport. Applied Mathematics Letters, 26(4):408-412.

Soares de Mello, J. C. C. B., Gomes, L. F. A. M., Gomes, E. G., and Soares de Mello, M. H. C. (2005). Use of ordinal multi-criteria methods in the analysis of the Formula 1 World Championship. Cadernos EBAPE.BR, 3(2):1-8.

Soares de Mello, J. C. C. B., Gomes Júnior, S. F., Angulo-Meza, L., and Mourão, C. L. d. O. (2015). Condorcet method with weakly rational decision makers: A case study in the Formula 1 Constructors' Championship. Procedia Computer Science, 55:493-502.

Szymanski, S. (2003). The economic design of sporting contests. Journal of Economic Literature, 41(4):1137-1187.

Szymanski, S. and Késenne, S. (2004). Competitive balance and gate revenue sharing in team sports. The Journal of Industrial Economics, 52(1):165-177.

Williams, C. and Crawford, G. (1980). Analysis of subjective judgment matrices. Interim report R-2572-AF, Rand Corporation, Santa Monica.

Zaksaitè, S. and Raduševičius, K. (2017). Manipulation of competitions in Formula1: where policy ends and cheating begins. The International Sports Law Journal, 16(3-4):240-246. 